\title{
MELANOMA IMAGE CLASSIFICATION SYSTEM BY NSCT FEATURES AND BAYES CLASSIFICATION
}

\author{
Sonia R, \\ Department of Information Technology, \\ JEPPIAAR SRR Engineering College, \\ Chennai, India \\ sonia.j25@gmail.com
}

\begin{abstract}
The knowledge obtained from a classification system is increasingly important for making a final decision. In this paper, a skin cancer classification system using Non-Sub sampled Contourlet Transform (NSCT) is presented. It uses double iterated filter banks to detect point discontinuities by a Laplacian pyramid and directional features by a directional filter bank. It allows the approximation of given image into a smooth contour at various level of decomposition. The Bayesian classifier is utilized in this work to classify the dermoscopic images in the PH2 database into normal or abnormal. From the results of the system, the melanoma image classification system can be used as a tool to make a final decision for the physicians.
\end{abstract}

Keywords: Melanoma Image Classification, Skin Cancer, NSCT, Bayes classifier.

\section{INTRODUCTION}

Skin cancer classification is a challenging task as there is no variation in the appearance of normal and lesions. Recently, many automated classification techniques are developed to classify the skin lesion using dermoscopic images. An automatic detection of gray areas that are specific to skin cancer is discussed in [1] using adaptive thresholding. It uses dermoscopic images to exactly identify the characteristics of the gray area by using the statistical histogram analysis in the multiple colour spaces. A skew estimation method is used to improve the adaptive thresholding results.

A CAD based melanoma system is discussed in [2]. Many types of features are extracted such as texture features using Discrete Wavelet Transform (DWT), border and geometric features using constructing a boundary-series model and the shape indexes respectively. Then, the gain-ratio method is used to select the optimized features, and the classification is done by Support Vector Machine (SVM), hidden naive Bayes, Random Forest (RF), and logistic model tree classifiers.

A computational dermoscopic image analysis system is discussed in [3]. A binary classification algorithm is used where the melanomas are classified into thin or thick. Skin cancer is diagnosed based on a technical characterization of a detailed micro-machined millimetre wave close to field probe in [4]. The broadband probe is optimized between 90 and $104 \mathrm{GHz}$ frequencies. It uses a tapered dielectric-rod waveguide which has metalized at the tip.

Early detection and prevention of melanoma using noninvasive real-time classification system are discussed in [5]. Skin burns by sun light are prevented by using real time alert. It consists of an image acquisition module followed by segmentation module to extract the lesion, feature extraction, and classification. The importance of diagnosing skin cancer by using an automated and computerized method is discussed in [6] using dermoscopic images. Statistical 
characteristics are used for the extraction of the pigment networks from the dermoscopic images.

A method of colour and textural features for the detection and classification of melanoma based on multilayer decomposition is discussed in [7]. Initially, the images are decomposed into a piecewise base layer by the weighted least squares framework. Then, the colour features from the colour histogram which is obtained from the base or smoothened layer. GLCM and the HOG symmetrical measures are taken as textural features and are classified using MSVM and the Extreme Learning Machine (ELM) classifiers.

A skin cancer screening system to classify melanoma images is discussed in [8]. Features are extracted using DWT by decomposing the input image and then, probabilistic neural network classifier is used to classify them. A therapeutic process that is used to identify the diseases at an early stage is discussed in [9]. An automatic melanoma recognition system by a self-advised learning and semi supervised model is discussed in [10]. An algorithm of self-advised SVM is used for enhancing the classification results of misclassified data. Then, a bootstrap method is used to increase the generalization capability of SA-SVMs.

A framework of 10 computer vision skin lesion features which includes texture and morphological features are extracted from 90 lesion images are discussed in [11]. In order to determine the important features, three types of algorithms such as; information gain measures, SVM recursive feature elimination, and correlation based feature subset selection methods are used. A method of early detection of melanoma is discussed in [12]. Features are extracted by the wavelet function and are classified using the SVM and neural network classifiers.

\section{MELANOMA IMAGE CLASSIFICATION SYSTEM}

The melanoma image classification system is shown in Figure 1. It has two stages; (1) feature extraction and (2) classification. The former module uses NSCT based transform algorithm for the extraction of features. Then, the extracted features of melanoma image are classified by the classifier known as the Bayes classification scheme. From the classification results, the performance of melanoma image classification system is measured.

\section{A. Feature Extraction by NSCT}

Feature extraction is the first module through which the specific feature values are extracted from the dermoscopic input images. The contourlet transform [13] uses double iterated filter banks to detect point discontinuities by a Laplacian pyramid and directional features by a directional filter bank. It allows the approximation of given image into a smooth contour at various level of decomposition. It provides multi-scale and directional features while decomposing the dermoscopic images at various scales.

As the contourlet transform provides up to $33 \%$ redundancy due to the down and up samplers present in both directional filter bank and Laplacian pyramid, NSCT is used. Figure 2 displays an overview of NSCT [13]. After decomposition by a predefined level, a number of sub-bands are generated. From the NSCT coefficients in each sub-band, energy features are extracted. 


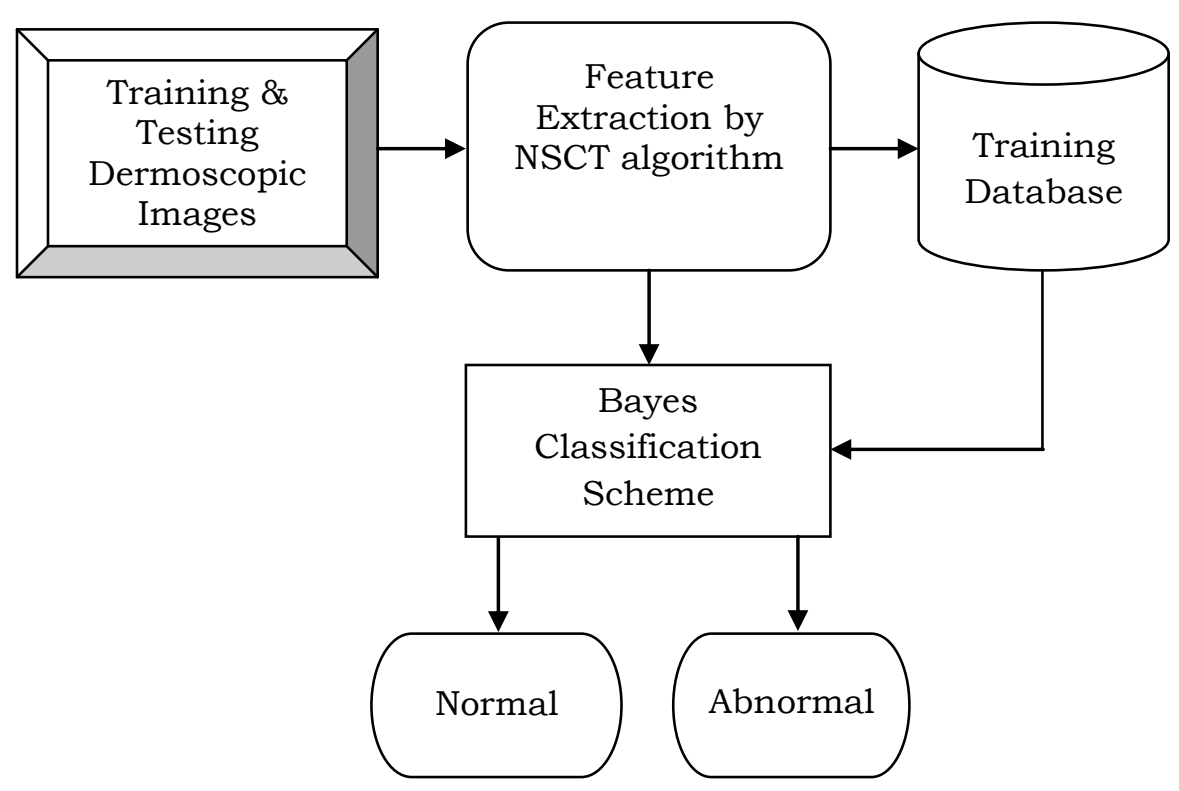

Fig. 1 Melanoma Classification system

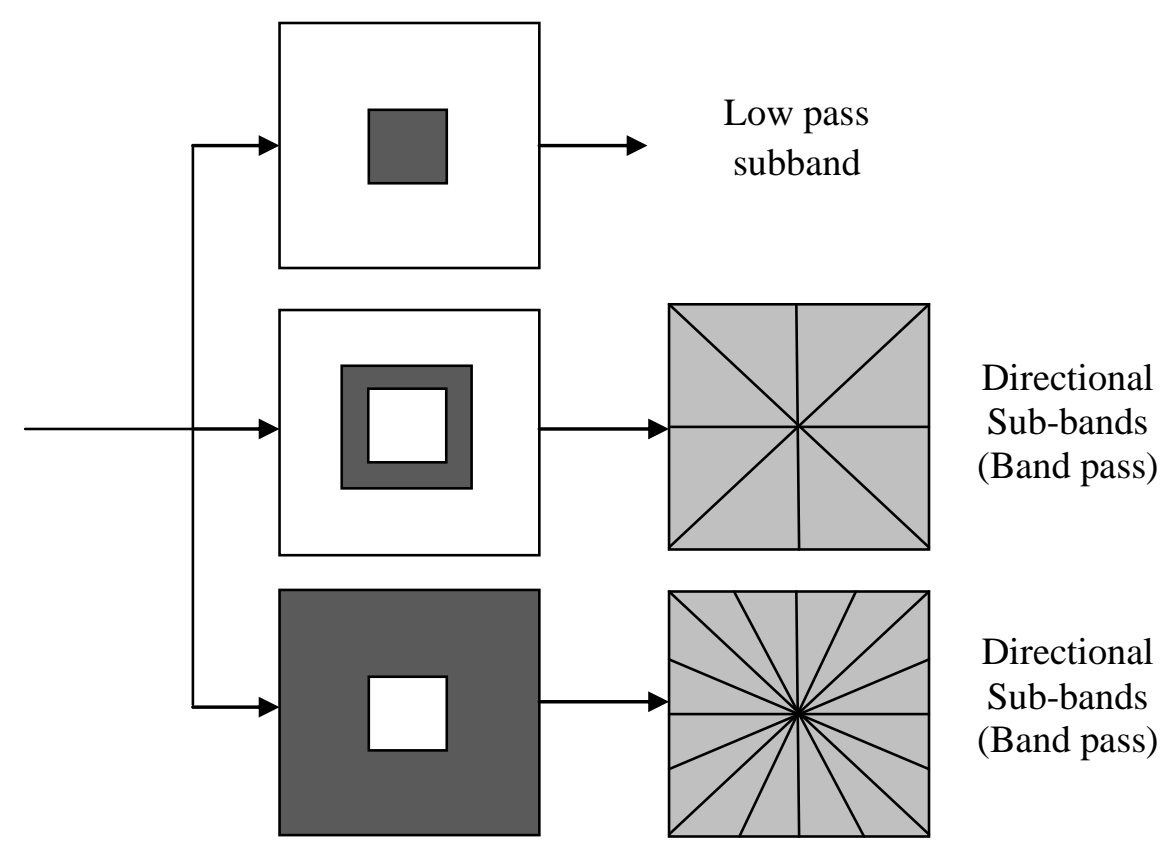

Fig. 2 Overview of NSCT

\section{B. Bayes Classification}

The mathematical modeling by Bayesian approach provides logical and rich backup. Also, it handles uncertainty very well and producing inferences by 
their well-defined procedures. The outcomes are rigorous and natural by combining prior and experimental information. A comprehensive framework is designed using the intimate relationship between decision theory and Bayesian statistics.

Let us consider the likelihood $p(y / \theta)$ and prior density $\pi(\theta)$ for a single observation $(y)$ related to $\theta$. For the same observation, the features of posterior distribution for $\theta$ can be explored by considering the posterior modes $\left(\theta^{*}\right)$. The differentiation of $y$ and $p$ in $\theta$ is defined in Eqn. 1.

$$
g_{\theta}\left(\theta^{*} \mid y\right)=0
$$

where

$$
\begin{aligned}
& g_{\theta}(\theta \mid y)=-\frac{\partial}{\partial \theta} \ln p(\theta \mid y) \\
& =-\frac{\partial}{\partial \theta} \ln \pi(\theta)-\frac{\partial}{\partial \theta} \ln p(y \mid \theta) \\
& =g_{\theta}(\theta)+g_{\theta}(y \mid \theta)
\end{aligned}
$$

where the efficient core function of likelihood $p(y / \theta)$ is $g_{\theta}(y \mid \theta)$. Eqn. 2 is known as the additive form of Bayes theory where the sum of likelihood score and prior score $\left(g_{\theta}(\theta)\right)$ is equal to the posterior score $g_{\theta}(y \mid \theta)$. It is very useful to analyze the behaviour of the posterior score in two ways: the variation of prior characteristics and the variation of single observation for a given likelihood.

\section{RESULTS AND DISCUSSION}

PH2 dermoscopic image database is employed in this study to analyze the performance of melanoma image classification system. PH2 database is maintained by Pedro Hispano hospital [14]. It consists of 120 dermoscopic images. There are 80 benign (normal) and 40 melanoma images available to the evaluation. All dermoscopic images are colour images of 8-bit depth. The size of each image is $768 \times 560$ pixels. Figure 3 and 4 shows a sample normal and abnormal dermoscopic images in the database respectively.
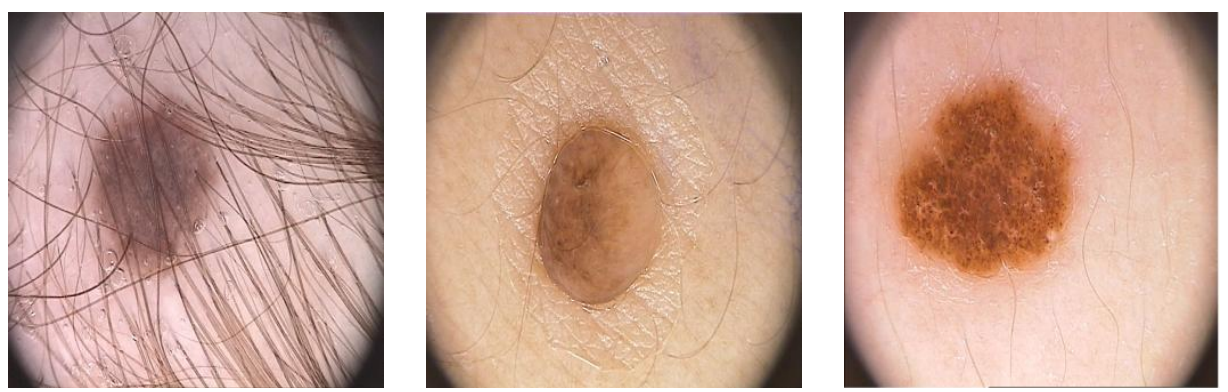
Fig. 3 Normal dermoscopic images in the PH2 database
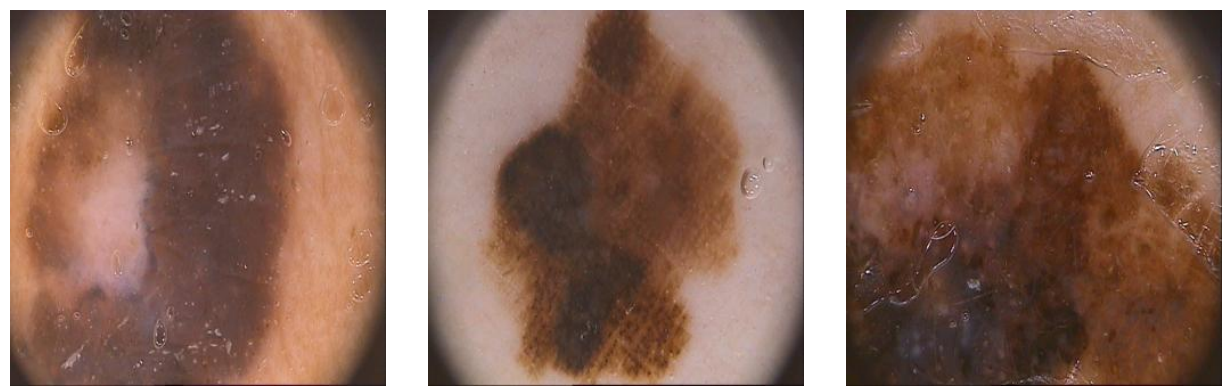

(b) Melanoma

Fig. 4 Abnormal dermoscopic images in the PH2 database

As it is a classification system, $\mathrm{k}$-fold cross validation (10-fold) is used. All images in a fold are used to test the melanoma classification system while the images in the remaining folds are used for training the classifier. Table 1 shows the overall classification performance metrics of melanoma classification system. Figure 5 shows the confusion matrix obtained using Bayes classifier.

\section{TABLE 1 Classification metrics of the melanoma classification}

\begin{tabular}{|c|c|c|}
\hline \multicolumn{3}{|c|}{ Performance measurement } \\
\hline Accuracy & Sensitivity & Specificity \\
\hline 96.7 & 97.5 & 96.3 \\
\hline
\end{tabular}

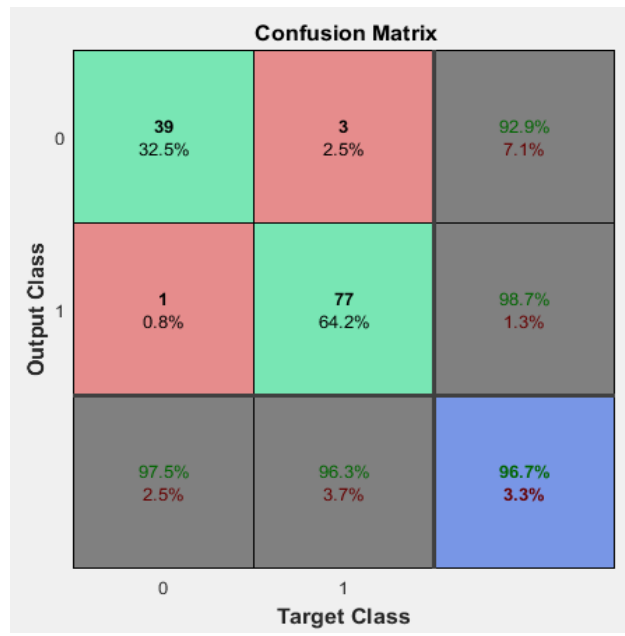

Fig. 5 Confusion Matrix of Melanoma image classification system

From the confusion matrix in Figure 3 , it is clear that the melanoma classification system gives $97.5 \%$ of sensitivity (only one melanoma image is misclassified as benign) and $96.3 \%$ of specificity ( 3 images are misclassified as malignant). The overall accuracy of the melanoma classification system is $96.7 \%$. 


\section{CONCLUSION}

An efficient analysis of NSCT for melanoma image classification is proposed in this paper. It used dermoscopic images as input data and classified into normal/abnormal by Bayes classifier. Features are extracted from the training images using NSCT. Bayes classification with 10-fold validation is used during classification. The effectiveness of melanoma image classification system is using PH2 database dermoscopic images. For all images in the database, the classification system generated a high classification accuracy of $96.7 \%$.

\section{REFERENCES}

[1]. G. Sforza, G. Castellano, S.A. Arika, R.W. LeAnder, R.J. Stanley, W.V. Stoecker, and J.R. Hagerty, "Using adaptive thresholding and skewness correction to detect gray areas in melanoma in situ images", IEEE Transactions on Instrumentation and Measurement, Vol. 61, No. 7, 2012, pp.1839-1847.

[2]. R. Garnavi, M. Aldeen, and J. Bailey, "Computer-aided diagnosis of melanoma using border-and wavelet-based texture analysis", IEEE Transactions on Information Technology in Biomedicine, Vol. 16, No. 6, 2012, pp. 1239-1252.

[3]. A. Saez, J. Sanchez-Monedero, P.A. Gutierrez, and C. Hervas-Martínez, "Machine learning methods for binary and multiclass classification of melanoma thickness from dermoscopic images", IEEE transactions on medical imaging, Vol. 35, No. 4, 2015, pp. 1036-1045.

[4]. F. Topfer, S. Dudorov, and J. Oberhammer, "Millimeter-wave near-field probe designed for high-resolution skin cancer diagnosis", IEEE Transactions on Microwave Theory and Techniques, Vol. 63, No. 6, 2015, pp. 2050-2059.

[5]. O. Abuzaghleh, B.D. Barkana, and M. Faezipour, "Noninvasive real-time automated skin lesion analysis system for melanoma early detection and prevention", IEEE journal of translational engineering in health and medicine, Vol. 3, 2015, pp. 1-12.

[6]. N. Alfed, F. Khelifi, A. Bouridane, and H. Seker, "Pigment network-based skin cancer detection", IEEE 37th Annual International Conference on Engineering in Medicine and Biology Society, 2015, pp. 7214-7217.

[7]. D. Choudhury, A. Naug, and S. Ghosh, "Texture and color feature based WLS framework aided skin cancer classification using MSVM and ELM", IEEE Annual India Conference, 2015, pp. 1-6.

[8]. Y.K. Jain, and M. Jain, "Skin cancer detection and classification by using Wavelet Transform and Probabilistic Neural Network", 2012, pp. 250-252.

[9]. A. Kardynal, and M. Olszewska, "Modern non-invasive diagnostic techniques in the detection of early cutaneous melanoma", Journal of dermatological case reports, Vol. 8, No. 1, 2014, pp. 1-8.

[10]. A. Masood, A. Al-Jumaily, and K. Anam, "Self-supervised learning model for skin cancer diagnosis", IEEE $7^{\text {th }}$ International IEEE/EMBS Conference on Neural Engineering, 2015, pp. 1012-1015. 
[11]. M. Mete, and N.M. Sirakov, "Optimal set of features for accurate skin cancer diagnosis", IEEE International Conference on Image Processing, 2014, pp. 2256-2260.

[12]. H.R. Mhaske, and D.A. Phalke, "Melanoma skin cancer detection and classification based on supervised and unsupervised learning", IEEE International conference on Circuits, Controls and Communications, 2013, pp. $1-5$.

[13]. N. Minh Do, and M. Vetterli, "The contourlet transform: An efficient directional multiresolution image representation," IEEE Transactions on Image Processing, IEEE Transactions on Image Processing, Vol. 14, 2004, pp. 2091-2106.

[14]. T. Mendonca, P.M. Ferreira, J.S. Marques, A.R. Marcal, and J. Rozeira, "PH 2-A dermoscopic image database for research and benchmarking", 35th Annual International Conference of the IEEE in Engineering in Medicine and Biology Society, 2013, pp. 5437-5440. 